\title{
Universiteit
}

Leiden

The Netherlands

\section{Polarization-switchable single photon source using the Stark effect}

Rakher, M.T.; Stoltz, N.G.; Coldren, L.A.; Petroff, P.M.; Bouwmeester, D.

\section{Citation}

Rakher, M. T., Stoltz, N. G., Coldren, L. A., Petroff, P. M., \& Bouwmeester, D. (2008). Polarization-switchable single photon source using the Stark effect. Applied Physics Letters, 93(9), 091118. doi:10.1063/1.2978396

Version: $\quad$ Not Applicable (or Unknown)

License: $\quad$ Leiden University Non-exclusive license

Downloaded from: https://hdl.handle.net/1887/65894

Note: To cite this publication please use the final published version (if applicable). 


\section{Polarization-switchable single photon source using the Stark effect}

M. T. Rakher, N. G. Stoltz, L. A. Coldren, P. M. Petroff, and D. Bouwmeester

Citation: Appl. Phys. Lett. 93, 091118 (2008); doi: 10.1063/1.2978396

View online: https://doi.org/10.1063/1.2978396

View Table of Contents: http://aip.scitation.org/toc/apl/93/9

Published by the American Institute of Physics

\section{Articles you may be interested in}

Invited Review Article: Single-photon sources and detectors

Review of Scientific Instruments 82, 071101 (2011); 10.1063/1.3610677

Single-mode solid-state single photon source based on isolated quantum dots in pillar microcavities Applied Physics Letters 79, 2865 (2001); 10.1063/1.1415346

Electrically driven and electrically tunable quantum light sources

Applied Physics Letters 110, 071102 (2017); 10.1063/1.4976197

Quantum interference of two photons emitted from a luminescence center in GaAs:N

Applied Physics Letters 110, 152102 (2017); 10.1063/1.4979520

Giant Stark effect in the emission of single semiconductor quantum dots

Applied Physics Letters 97, 031104 (2010); 10.1063/1.3460912

Control of polarized single quantum dot emission in high-quality-factor microcavity pillars

Applied Physics Letters 88, 051113 (2006); 10.1063/1.2171803

\section{Conference Proceedings}

Get $30 \%$ off all print proceedingst

Enter Promotion Code $P D=30$ at checkout 


\title{
Polarization-switchable single photon source using the Stark effect
}

\author{
M. T. Rakher, ${ }^{1, a)}$ N. G. Stoltz, ${ }^{2}$ L. A. Coldren, ${ }^{2,3}$ P. M. Petroff, ${ }^{2,3}$ and D. Bouwmeester ${ }^{1,4}$ \\ ${ }^{1}$ Department of Physics, University of California Santa Barbara, Santa Barbara, California 93106, USA \\ ${ }^{2}$ Materials Department, University of California Santa Barbara, Santa Barbara, California 93106, USA \\ ${ }^{3}$ ECE Department, University of California Santa Barbara, Santa Barbara, California 93106, USA \\ ${ }^{4}$ Huygens Laboratory, Leiden University, P.O. Box 9504, 2300 RA Leiden, The Netherlands
}

(Received 5 June 2008; accepted 18 August 2008; published online 5 September 2008)

\begin{abstract}
A polarization-switchable single photon source is demonstrated by embedding a self-assembled quantum dot in a high-quality, electrically gated, oxide-apertured micropillar cavity. Due to the noncircular aperture, the polarization degeneracy of the fundamental cavity mode is lifted, leaving two linearly polarized $Q \approx 20000$ modes separated by $194 \mu \mathrm{eV}$. An intracavity electric field generated by an applied bias enables Stark shift tuning of the quantum dot emission over a frequency range containing both polarization modes, switching the dominant single photon polarization through the Purcell effect. We measure polarization switching up to $300 \mathrm{kHz}$, limited by the $R C$ time constant of the device. (C) 2008 American Institute of Physics.
\end{abstract}

[DOI: $10.1063 / 1.2978396]$

Self-assembled quantum dots (QDs) embedded in microcavities have been used to demonstrate a range of novel optical devices with potential for future technologies. To date, this has included bright sources of single photons, ${ }^{1,2}$ ultralow threshold lasers, ${ }^{3}$ and cavity quantum electrodynamic (QED) devices. ${ }^{4-6}$ One of the advantages of the QD microcavity system is that the QD behaves like an atom that is permanently trapped in its semiconductor matrix, negating the use of trapping lasers as in atomic cavity QED systems. ${ }^{7,8}$ The disadvantage of using QDs is that they are not identical like atoms. Because of this fact, the QD microcavity system must take advantage of an external control to achieve spectral resonance. To date, this has been done by shifting the QD with temperature ${ }^{4,5}$ or by shifting the microcavity mode by depositing a small amount of material onto the structure. ${ }^{9,10}$ In principle, the QD energy could be tuned with an electric field by the quantum confined Stark effect. ${ }^{11,12}$ In this letter, the Stark effect is used to demonstrate a novel single photon device where a QD embedded in an oxide-apertured micropillar cavity is electrically tuned into resonance with two orthogonally polarized cavity modes, creating a polarizationswitchable single photon source.

The types of cavities used in the experiments are oxideapertured micropillars. ${ }^{13,14}$ In these micropillars, optical confinement in the growth direction is provided by two distributed Bragg reflector (DBR) stacks made of alternating layers of GaAs and $\mathrm{Al}_{0.9} \mathrm{Ga}_{0.1}$ As. Lateral confinement is provided by the index contrast between the unoxidized AlAs and the $\mathrm{AlO}_{x}$ in the aperture region. This type of gentle confinement has been shown to achieve a quality factor $Q$ of up to $48000 .{ }^{14}$ Additionally, the aperture can be engineered to be noncircular, for example, by exploiting the fact that the oxidation proceeds at different rates along different crystalline axes. ${ }^{15}$ The ellipticity of the aperture breaks the polarization degeneracy of the fundamental $\mathrm{HE}_{1, \pm 1}$ mode, ${ }^{2,16}$ leaving two linearly polarized modes with small energy splitting, as shown in Fig. 1. In the cavity considered here, $Q$ s were 18920 and 19320 for the horizontally and vertically polarized modes, respectively; the polarization visibility was 0.95

${ }^{a)}$ Electronic mail: rakher@physics.ucsb.edu. for each mode and the mode splitting $\delta$ was $194 \mu \mathrm{eV}$.

Samples are grown by molecular beam epitaxy on a semi-insulating GaAs [100] substrate. There are four sections in the structure, the bottom mirror, the active region, the oxide-aperture region, and the top mirror as described in more detail in a previous study. ${ }^{14}$ The active region contains a centered InAs QD layer grown in the Stranski-Krastanov growth mode. The optical GaAs cavity is $\mathrm{Si} n$-doped up to $20 \mathrm{~nm}$ above the single layer of QDs providing a back gate. The first GaAs DBR period in the bottom mirror is C $p$-doped providing a top gate. Two etch windows with selective countersink depth are created using reactive ion etch (RIE) in $\mathrm{Cl}_{2}$ plasma away from the cavities and contacted using a Ti/Pt/Au metallization. Cavity trenches were formed by optical lithography and RIE penetrating approximately five mirror periods into the bottom DBR. This etches small trenches in the surface that are used to define the $\mathrm{AlO}_{x}$ oxi-

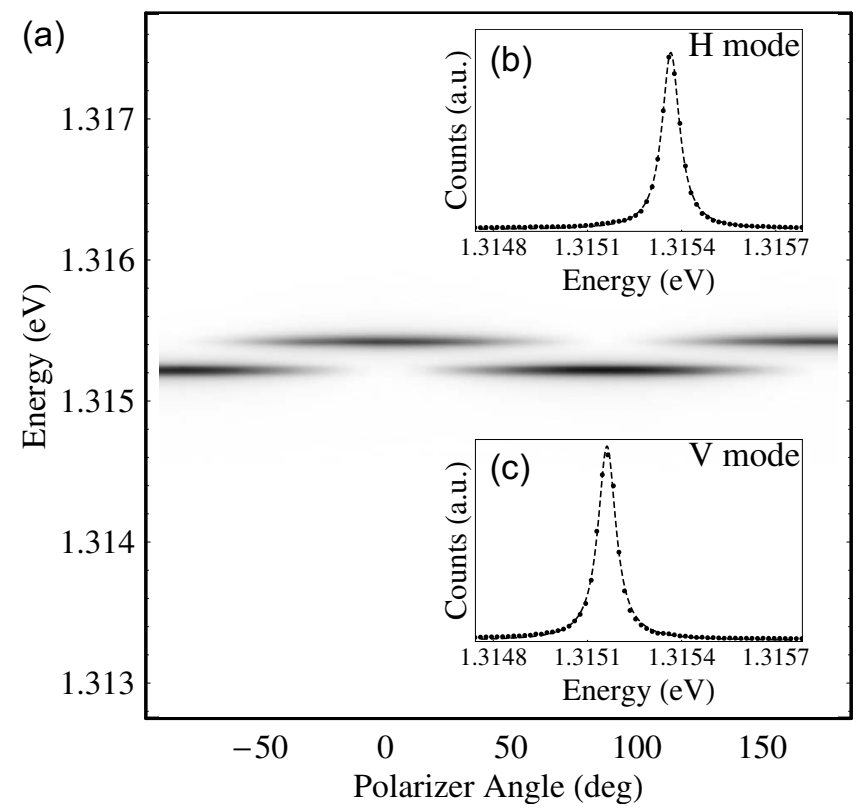

FIG. 1. (a) The spectra of the nondegenerate fundamental modes as a function of polarizer angle. [(b)-(c)] The spectra of the $H$ and $V$ modes, respectively, with Lorentzian fits yielding $Q=18920$ and $Q=19320$. 

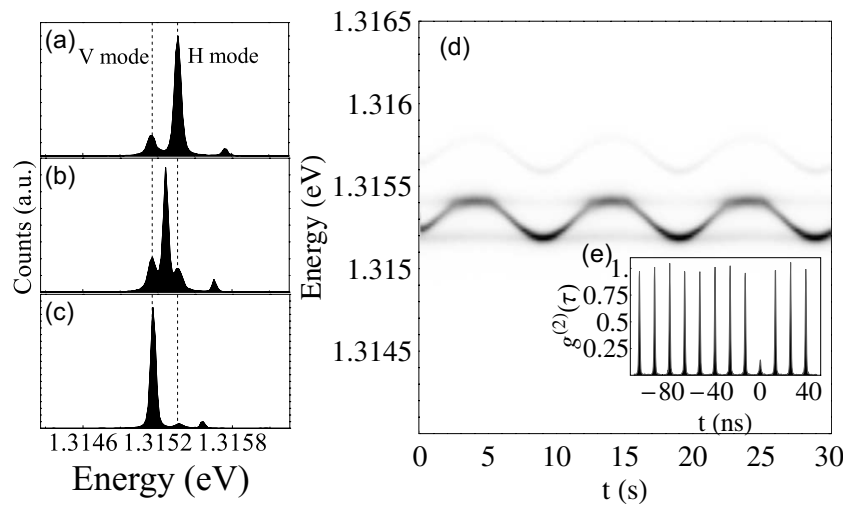

FIG. 2. [(a)-(c)] The spectra for applied biases of 7.62, 8.33, and $8.86 \mathrm{~V}$, showing the QD in resonance with the $H$ mode, in between both modes, and in resonance with the $V$ mode. (d) The spectra as a function of time with a $100 \mathrm{mHz}$ sinusoidal driving bias. (e) The second order correlation function $g^{(2)}(\tau)$ for the QD in resonance with the $H$ mode.

dation front. Tapered $\mathrm{AlO}_{x}$ apertures are formed laterally during steam oxidation within the cavity area creating inner openings of $0.5-2 \mu \mathrm{m}$. The doped layers enable electrical gating of the sample that in turn creates an adjustable electric field over the QD region. This external field causes the QD emission to spectrally shift, a phenomena known as the quantum confined Stark effect. ${ }^{11}$

All measurements are performed in a He-flow cryostat at $4.2 \mathrm{~K}$. Microphotoluminescence spectroscopy is implemented by focusing $500 \mathrm{nW}$ of $860 \mathrm{~nm}$ laser through a highpower (numerical aperture of 0.42 ) objective onto the sample surface. The emission is then directed to a spectrometer equipped with a charge coupled device (CCD) array, yielding a spectral resolution of $30 \mu \mathrm{eV}$ or to two avalanche photodiodes (APDs) for single photon correlation measurements. The sample is electrically connected to a function generator capable of operating up to $50 \mathrm{MHz}$. As shown in the device in Figs. 2(a)-2(c), emission from a QD can be electrically tuned into resonance with the modes from Fig. 1. Near 7.62 $\mathrm{V}$, the QD emission is in resonance with the $H$-mode. Because of the Purcell effect, QD emission into this mode is enhanced by the Purcell factor $F_{p}$, while $V$-polarized emission is inhibited by a factor denoted as $\Gamma_{\text {in }}$. As a result, the emission is dominantly $H$-polarized with a ratio of $F_{p} / \Gamma_{\text {in }}$. Similarly, at $8.86 \mathrm{~V}$, the emission is in resonance with the $V$-mode and is dominantly $V$-polarized. In this way, the polarization of the single photons emitted by the QD can be electrically switched between dominantly $H$ and $V$. Figure 2(d) shows the spectra as a function of time with a $100 \mathrm{mHz}$ sinusoidal drive bias demonstrating the principle of polarization switching. This frequency was chosen in order to provide a sufficient drive frequency to the sampling frequency ratio of the CCD array. The QD emission clearly oscillates at the drive frequency between the two modes, emitting single photons whose polarization are switched at the same drive frequency. In addition, another off-resonant QD can be seen slightly higher in energy and spectrally oscillates at the drive frequency. For the pump powers used here, the second order correlation function $g^{(2)}(\tau)$ always has a value $g^{(2)}(0)$ $<0.15$, as shown in Fig. 2(e), well below the minimum of 0.5 required to prove a single photon operation. Note that in comparison to the QD Stark effect literature, ${ }^{11}$ the applied biases used here are much larger. This is due to large contact resistances but based on the amount of spectral tuning, the
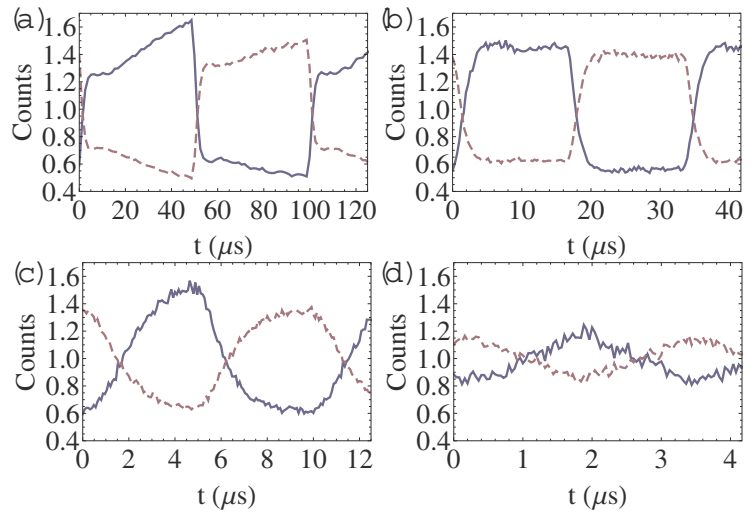

FIG. 3. (Color online) [(a)-(d)] Normalized and time-integrated APD counts for switching at drive frequencies of 10,30,100, and $300 \mathrm{kHz}$. $H(V)$-polarization is denoted in dashed magenta (straight blue).

electric field at the QDs is approximately $50 \mathrm{kV} / \mathrm{cm}$ and varies by approximately $40 \mathrm{kV} / \mathrm{cm}$.

To probe the quality of the polarization switching and to find its temporal limit, we measured switching as a function of drive frequency. This was accomplished by using a dc bias to place the QD emission spectrally equidistant from each mode, and then applying an additional ac square wave to oscillate the QD. The amplitude of the square wave was set so that the QD could reach both modes at low frequency. $H$ and $V$ polarized photons were separated at a polarizing beamsplitter and each detected at an APD. The signal from the APDs was sent to a multichannel analyzer triggered by the function generator. Normalized integrated photon counts as a function of time for each polarization are shown in Fig. 3 for drive frequencies of $10,30,100$, and $300 \mathrm{kHz}$ at constant drive amplitude with $H(V)$ detection events in dashed magenta (straight blue). As can be seen clearly in the $10 \mathrm{kHz}$ measurements, the signals have both a slow and fast temporal component, with the slow component vanishing for the higher frequency measurements. This will be explored in detail in the next section.

For a given drive frequency, the quality of the polarization switching can be quantitatively described by the average of the visibilities of the count rate oscillations, as shown in Fig. 3, where the visibility is defined as $V_{p} \equiv\left(I_{\max }\right.$ $\left.-I_{\min }\right) /\left(I_{\max }+I_{\min }\right)$ for each polarization measurement. Figure 4 summarizes the average visibility as a function of drive frequency with constant drive amplitude. Because the system has a limited frequency response, the visibility is expected to drop off once the frequency cutoff is surpassed and the amplitude of the spectral oscillation of the QD is not large

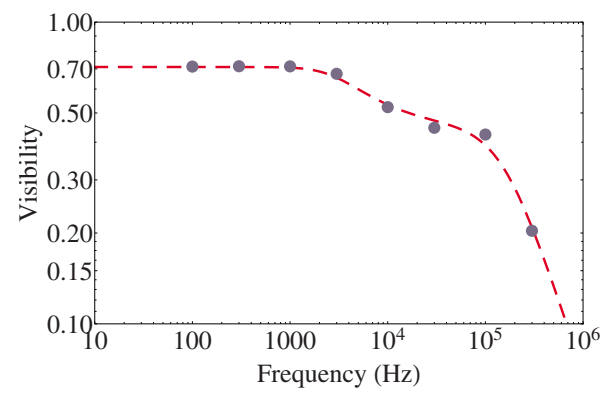

FIG. 4. (Color online) The average polarization visibility as a function of drive frequency with constant amplitude. A fit to the data using Eqs. (1) and (2) yields a thermal cutoff frequency of $14 \pm 4.1 \mathrm{kHz}$ and an $R C$ cutoff frequency of $1.09 \pm 0.10 \mathrm{MHz}$. 
enough to reach the modes. As Fig. 4 clearly shows, there are two cutoff frequencies, one around $10 \mathrm{kHz}$ and another near $1 \mathrm{MHz}$. This was expected as two temporal components were visible in Fig. 3(a). We hypothesize and later verify that the lower frequency cutoff corresponds to the current-induced heating of the sample and the higher frequency corresponds to the $R C$ electrical cutoff. To model the dependence of the visibility on drive frequency, the emission intensity of each polarization as a function of QD spectral position must be known. This dependence can be well approximated by a Lorentzian with width (height) determined by the $Q$-factor (Purcell factor $F_{p}$ ) and a constant background due to the inhibited decay into modes other than the cavity $\Gamma_{\text {in }}$. The average visibility can then be expressed as

$V_{p}=\frac{4 \Delta \delta \kappa^{2} F_{p}}{\Gamma_{\text {in }}\left(\delta^{2}-4 \Delta^{2}\right)^{2}+\left(F_{p}+2 \Gamma_{\text {in }}\right)\left(\delta^{2}+4 \Delta^{2}\right) \kappa^{2}+\left(\Gamma_{\text {in }}+F_{p}\right) \kappa^{4}}$,

where $\Delta$ is the QD spectral tuning amplitude, $\delta$ is the mode splitting, and $\kappa$ is the mode linewidth. $\kappa$ and $\delta$ are known from the optical spectrum (Fig. 1) and $F_{p}$ was measured by time-resolved spectroscopy to be approximately 5 for both modes. $\Gamma_{\text {in }}$ can be determined as follows. At a sufficiently low drive frequency the QD spectrally reaches both modes so $\Delta=\delta / 2$. This value of $\Delta$ gives the highest possible visibility for this device and is measured to be $0.71 \pm 0.007$, determining $\Gamma_{\text {in }}$ to be $(0.85 \pm .03) \%$ of the bulk decay rate. The maximum switching visibility is less than the pure mode polarization visibility of 0.95 because of $F_{p}$ and $\Gamma_{\text {in }}$.

The frequency dependence of the QD spectral tuning amplitude $\Delta=\Delta(\nu)$ can be described as the sum of two incoherent processes, each with a specific cutoff frequency. This function is expressed as

$$
\Delta(\nu)=\frac{\delta / 2}{A_{T}+1}\left[\frac{\nu_{R C}}{\sqrt{4 \pi^{2} \nu^{2}+\nu_{R C}^{2}}}+\frac{\nu_{T} A_{T}}{\sqrt{4 \pi^{2} \nu^{2}+\nu_{T}^{2}}}\right],
$$

where $\nu_{T}\left(\nu_{R C}\right)$ is the thermal $(R C)$ cutoff frequency, and $A_{T}$ is the relative strength of the thermal process. By substituting Eq. (2) into Eq. (1), the visibility data can be fit. The curve with best fit parameters is shown in Fig. 4. From this fit, we determine the thermal cutoff frequency $\nu_{T}$ to be $14 \pm 4.1 \mathrm{kHz}$ and the $R C$ cutoff frequency, $\nu_{R C}$ to be $1.1 \pm 0.10 \mathrm{MHz}$. A simple estimate of the thermal cutoff using the low-temperature specific heat and thermal conductivity of GaAs, as well as the device size, yields a value of approximately $10 \mathrm{kHz}$, which is in agreement with the fit. Furthermore, additional low-temperature capacitance-voltage measurements were performed to verify the origin of the high frequency cutoff. The resistance and capacitance near the working voltages of the experiment were found to be $1.7 \mathrm{k} \Omega$ and $0.49 \mathrm{nF}$, respectively, yielding an $R C$ frequency cutoff of $1.2 \mathrm{MHz}$, in agreement with the fit.

In conclusion, we have demonstrated a method for onchip, single photon polarization switching by Stark tuning emission from a single QD into resonance with two, highquality, orthogonally polarized cavity modes. While our demonstration was temporally limited by the thermal conductivity of the device and ultimately by the $R C$ cutoff, future implementations can be made faster reducing the current and by decreasing the contact resistance and parasitic capacitance. In principle, switching rates should be able to approach gigahertz frequencies, similar to recent results on fast
QD charging. ${ }^{17,18}$ Additionally, the polarization visibility was limited in this case to 0.71 by $F_{p}$ and $\Gamma_{\text {in. }}$. The visibility could be increased to 0.96 if $\Gamma_{\text {in }}$ and $F_{p}$ had values like those found in photonic crystal defect cavities ${ }^{3}$ and the mode splitting was increased by a factor of 2 . The combination of these advances will enable high visibility switching at gigahertz frequencies. This kind of switching could enable high frequency lock-in measurements of single QDs (Ref. 17) or fast loading of a cavity for bias-controlled optical nonlinearities in cavity QED. ${ }^{19-21}$ Furthermore, this type of single photon polarization switching could have applications as an excitation mechanism for other quantum systems or for classical optical communication.

This work was supported by the National Science Foundation NIRT (No. 0304678), DARPA (No. MDA 972-01-10027), and Marie-Curie (No. EXT-CT-2006-042580).

${ }^{1}$ M. Pelton, C. Santori, J. Vučković, B. Zhang, G. S. Solomon, J. Plant, and Y. Yamamoto, Phys. Rev. Lett. 89, 233602 (2002).

${ }^{2}$ S. Strauf, N. G. Stoltz, M. T. Rakher, L. A. Coldren, P. M. Petroff, and D. Bouwmeester, Nat. Photonics 1, 704 (2007).

${ }^{3}$ S. Strauf, K. Hennessy, M. T. Rakher, Y.-S. Choi, A. Badolato, L. C. Andreani, E. L. Hu, P. M. Petroff, and D. Bouwmeester, Phys. Rev. Lett. 96, 127404 (2006).

${ }^{4}$ T. Yoshie, A. Scherer, J. Hendrickson, G. Khitrova, H. M. Gibbs, G. Rupper, C. Ell, O. B. Shchekin, and D. G. Deppe, Nature (London) 432, 200 (2004).

${ }^{5}$ J. P. Reithmaier, G. Sęk, A. Löffler, C. Hofmann, S. Kuhn, S. Reitzenstein, L. V. Keldysh, V. D. Kulakovskii, T. L. Reinecke, and A. Forchel, Nature (London) 432, 197 (2004).

${ }^{6}$ K. Hennessy, A. Badolato, M. Winger, D. Gerace, M. Atatüre, S. Gulde, S. Fält, E. L. Hu, and A. Imamoğlu, Nature (London) 445, 896 (2007).

${ }^{7}$ S. J. van Enk, J. McKeever, H. J. Kimble, and J. Ye, Phys. Rev. A 64, 013407 (2001).

${ }^{8}$ J. McKeever, J. R. Buck, A. D. Boozer, A. Kuzmich, H.-C. Nägerl, D. M. Stamper-Kurn, and H. J. Kimble, Phys. Rev. Lett. 90, 133602 (2003).

${ }^{9}$ S. Mosor, J. Hendrickson, B. C. Richards, J. Sweet, G. Khitrova, H. M. Gibbs, T. Yoshie, A. Scherer, O. B. Shchekin, and D. G. Deppe, Appl. Phys. Lett. 87, 141105 (2005).

${ }^{10}$ S. Strauf, M. T. Rakher, I. Carmeli, K. Hennessy, C. Meier, A. Badolato, M. J. A. Dedood, P. M. Petroff, E. L. Hu, E. G. Gwinn, and D. Bouwmeester, Appl. Phys. Lett. 88, 043116 (2006).

${ }^{11}$ P. W. Fry, I. E. Itskevich, D. J. Mowbray, M. S. Skolnick, J. J. Finley, J. A. Barker, E. P. O'Reilly, L. R. Wilson, I. A. Larkin, P. A. Maksym, et al., Phys. Rev. Lett. 84, 733 (2000).

${ }^{12}$ F. Hofbauer, S. Grimminger, J. Angele, G. Böhm, R. Meyer, M. C. Amann, and J. J. Finley, Appl. Phys. Lett. 91, 201111 (2007).

${ }^{13} \mathrm{C}$. Wilmsen, H. Temkin, and L. A. Coldren, Vertical-Cavity SurfaceEmitting Lasers (Cambridge University Press, Cambridge, England, 1999).

${ }^{14}$ N. G. Stoltz, M. Rakher, S. Strauf, A. Badolato, D. D. Lofgreen, P. M. Petroff, L. A. Coldren, and D. Bouwmeester, Appl. Phys. Lett. 87, 031105 (2005).

${ }^{15}$ A. Mizutani, N. Hatori, N. Nishiyama, F. Koyama, and K. Iga, IEEE Photonics Technol. Lett. 10, 633 (1998).

${ }^{16}$ D. C. Unitt, A. J. Bennett, P. Atkinson, D. A. Ritchie, and A. J. Shields, Phys. Rev. B 72, 033318 (2005).

${ }^{17}$ B. D. Gerardot, S. Seidl, P. A. Dalgarno, R. J. Warburton, M. Kroner, K. Karrai, A. Badolato, and P. M. Petroff, Appl. Phys. Lett. 90, 221106 (2007).

${ }^{18}$ M. Geller, A. Marent, T. Nowozin, D. Bimberg, N. Akçay, and N. Öncan, Appl. Phys. Lett. 92, 092108 (2008).

${ }^{19}$ A. Imamoglu, H. Schmidt, G. Woods, and M. Deutsch, Phys. Rev. Lett. 79, 1467 (1997).

${ }^{20}$ K. M. Birnbaum, A. Boca, R. Miller, A. D. Boozer, T. E. Northup, and H. J. Kimble, Nature (London) 436, 87 (2005).

${ }^{21}$ B. Dayan, A. S. Parkins, T. Aoki, E. P. Ostby, K. J. Vahala, and H. J. Kimble, Science 319, 1062 (2008). 